\title{
Exploration of the Barriers and Education Needs of Non-Pediatric Hospital Emergency Department Providers in Pediatric Trauma Care
}

\author{
Tricia Falgiani $^{1}$, Christopher Kennedy ${ }^{2}$, Sara Jahnke ${ }^{3}$ \\ ${ }^{1}$ Department of Emergency Medicine, Division of Pediatric Emergency Medicine, University of Florida, Gainesville, USA; ${ }^{2}$ Division \\ of Pediatric Emergency Medicine, The Children’s Mercy Hospital, Kansas City, USA; ${ }^{3}$ Institute for Biobehavioral Health Research, \\ National Development and Research Institutes, Leawood, USA. \\ Email: $\underline{\text { falgiani@ufl.edu }}$
}

Received October $30^{\text {th }}, 2013$; revised November $20^{\text {th }}, 2013$; accepted December $18^{\text {th }}, 2013$

Copyright (C) 2014 Tricia Falgiani et al. This is an open access article distributed under the Creative Commons Attribution License, which permits unrestricted use, distribution, and reproduction in any medium, provided the original work is properly cited. In accordance of the Creative Commons Attribution License all Copyrights (c) 2014 are reserved for SCIRP and the owner of the intellectual property Tricia Falgiani et al. All Copyright (C) 2014 are guarded by law and by SCIRP as a guardian.

\section{ABSTRACT}

Study Objective: Nine million children are seen in emergency departments each year for traumatic injuries. Eighty percent of these children will be cared for in non-children's hospital settings. We sought to understand the barriers and opportunities for optimal pediatric trauma care in non-pediatric emergency departments and to define practice-specific educational needs. Methods: This qualitative study consisted of focus groups from rural, regional and urban non-pediatric emergency department sites discussing pediatric trauma care. Groups were homogenous for the provider role and included 8 physician groups and 9 non-physician groups. Focus groups were led by a trained moderator using a discussion guide composed of open-ended questions which covered various topics of pediatric trauma care. Focus groups were audio-taped and later transcribed and the data were analyzed for major themes and key concepts. Results: A total of 107 providers participated in the focus groups (32 physicians and 75 non-physicians). Barriers to provide optimal pediatric trauma care expressed by providers included the lack of pediatric trauma experience, inadequate pediatric trauma training and the lack of confidence with assessment of the pediatric trauma patient. All providers across all types of hospitals indicated a need and interest in training focused on pediatric trauma, but topics covered, and skills needed varied by type of facility. Conclusions: Community emergency room providers indicated a need for pediatric trauma education. Specifically, hands-on training with high-fidelity simulation was identified as the most useful type of training to gain the skills and confidence needed to manage pediatric trauma patients in their emergency departments.

\section{KEYWORDS}

Pediatric Trauma; Education; High Fidelity Simulation; Community Emergency Department

\section{Introduction}

\subsection{Background}

More than nine million children are seen in emergency departments (EDs) each year for traumatic injuries [1]. One recent study estimated that over 17 million children live more than an hour away from the nearest pediatric trauma center [2]. It is important to recognize that eighty percent of children will be cared for in non-children's hospital settings [3]. Despite the frequency of childhood injuries, many healthcare providers practice in settings where treating a critically injured child is rare. Resuscitation skills may degrade if not well rehearsed and provider confidence is diminished in unfamiliar situations.

There are many differences in anatomy, physiology and developmental level in children that range from the simple difference of the diameter of the growing airway to the complex emotional development between late childhood and early adolescence. These distinctions directly impact the care delivered to children as well as the 
manner in which it is delivered. Providers unfamiliar with the nuances of anatomical, physiological, and emotional development may feel considerable trepidation when faced with a critically injured child.

Recognizing the need for improvement in the training and education of health care professionals that treat children, the 2006 Institute of Medicine (IOM) report Emergency Care for Children: Growing Pains putting forth the following recommendation [4]:

That Every pediatric- and emergency care-related health professional credentialing and certification body define pediatric emergency care competencies and require practitioners to receive the level of initial and continuing education necessary to achieve and maintain those competencies.

During the past thirty years efforts to improve care have focused on equipment and protocols, but large gaps still remain. There is a paucity of research in the area of pediatric trauma care education, and even less in community emergency departments. Ideally, children with traumatic injuries should be treated by pre-hospital and emergency department providers that are both educated and experienced in pediatric trauma care. To design an educational intervention that incorporates both the providers' needs and pediatric trauma care principles requires an understanding of the opportunities and barriers to care for injured children in community emergency departments.

\subsection{Importance}

The severely injured child demands prompt medical interventions from health care providers. In order to develop a pediatric trauma educational program and improve the care for pediatric trauma patients in community emergency departments, we first must understand the environments, resources, experiences, circumstances and barriers to care and education that community providers face. All too often programs are developed without a comprehensive understanding of the providers' views and team dynamics. The knowledge gained from this study aims to improve the care of injured children through the careful development of educational programs designed to meet the specific needs of providers in community emergency departments.

\subsection{Goals of This Investigation}

The purpose of this study is to understand the barriers and opportunities for optimal community pediatric trauma care in urban, regional and rural emergency departments and to define areas of educational needs specific to each type of emergency department. Using qualitative methodology, we conducted a series of focus groups with physicians, physician extenders, nurses, EMS personnel and ED support staff.

\section{Methods}

\subsection{Study Design}

Seventeen separate focus groups were conducted in nonpediatric community emergency department sites. Focus groups can provide important insight into current practice and help identify potential areas for improving current practices and processes by examining the opinions, experiences and attitudes of participants [5]. The study was reviewed and determined to be exempt from formal review by the institutional review board.

\subsection{Setting and Selection of Participants}

This study took place from July 2009 through February 2010 in 3 urban, 3 regional and 4 rural hospitals. Participants were recruited by the coordinator of the Area Health Education Centers by electronic, verbal and posted announcements in the workplace. Participants included any hospital or pre-hospital based practitioner who cares for children, including but not limited to, full or part-time physicians, nurse practitioners, physician assistants, nurses, nursing managers, EMTs, paramedics, flight nurses, and respiratory therapists. To eliminate concern for power differential, focus groups were separated into two groups: 1) nurses, respiratory therapists, paramedics/EMTs and other ED staff members and 2) physicians, physician assistants, nurse practitioners. Participation was completely voluntary and participants were not compensated. A total of 104 providers participated in the focus groups, of which 29 were physician and physician extenders and 75 were nursing, EMS and other ED staff members.

\subsection{Methods of Measurement}

In order to ensure that a diverse sample of hospital and emergency department staff were included in the data collection, sites were recruited from three different types of hospitals: urban, regional and rural. Focus groups began with an introduction of the research team, ground rules and an explanation of the group process. Groups were homogenous for provider role and included 8 physician/physician extender groups and 9 non-physician provider groups. The groups were conducted using a semi-structured format led by a trained moderator who directed and facilitated discussion using a discussion guide composed of open-ended questions. The moderator guided participants through specific topic areas including pediatric trauma experience; barriers to pediatric trauma care; emergency department team dynamics; training and 
education. Each group lasted approximately sixty minutes. Each session was audio-taped by two electronic recorders and notes were taken for non-verbal cues as well as clarification of audio. The audio-tapes were transcribed and the data analyzed for major themes and key concepts with the overall purpose of creating a sophisticated and rich description of beliefs, opinions and perceptions related to the experience and management of pediatric trauma in general emergency departments.

\subsection{Primary Data Analysis}

The data for this study consisted of multiple focus group transcripts. First, researchers independently reviewed the transcribed documents to develop a familiarity with the text and to begin a thematic analysis by searching for themes that occurred frequently in a single focus group or were common across groups. Second, the data was coded by identifying passages that exemplified the key concepts and ideas related to the major themes. The use of multiple reviewers (Drs. Falgiani, Kennedy and Jahnke) assisted in establishing the construct validity and interrater reliability of the coding scheme and the identified codes. Agreement was reached on the coded themes. Finally the results were stratified by the type of hospital (urban, regional, rural) and similarities and differences between types of hospitals were noted.

\section{Results}

\subsection{Experiences with Pediatric Trauma}

Participants identified many barriers and challenges they face in pediatric trauma care in their facilities. In general, participants from all hospital types identified the rarity of pediatric trauma patients seen in their emergency departments as an impetus for increased training, as many felt out of practice. One urban physician stated, "You know, I think because we don't see that many pediatric traumas, I actually think we need even more training. Because, with adults, you see this stuff all the time and you're constantly refreshing yourself. If you get a little bit of training with pediatrics and then you don't see anything for six months or eight months, then you see something, it's like, 'Boy, what do I remember from way back then?' Um, I think it takes more effort to stay up to date with something that you're not exposed to a lot."

\subsection{Emergency Department Team Dynamics}

Providers in rural hospital environments indicated a lack of resources in providing pediatric trauma care. This included not having physicians in the emergency department at all times and having to call staff in from home in the event of a pediatric trauma. Also, in rural and region- al emergency departments there were not specifically designated trauma teams. Most of the providers that responded to pediatric trauma patients were general emergency department physician or physician extenders, nursing and support staff. Often times EMS personnel would also stay in the emergency department to help with the patient. A rural nurse said, "Our nurses are floor nurses, ER nurses, whatever they're doing. If they come in by EMS, you've got your EMS people there to help but if they don't, if they come in by car, you just have nursing, that's all you have. You're hope is that maybe one of them has a little more education in pediatrics and starting IVs and doing stuff to them $\cdots$ at least get that level done. But, you know, we don't really have assigned teams for really anything." A rural physician commented, "We have limited numbers, it is normally who is on call for the ER and if there is back up people, and the emergency room folks that are working or the night shift at the hospital. There is not a clear distinction between the ER and the inpatient in our facility. I mean, it is a small enough facility that sort of $\cdots$ so all the people that are covering inpatient are also covering the ER.”

Often they felt like the function of the hospital team was chaotic and underprepared to handle pediatric patients, especially in the event of a life-threatening injury.

Alternatively, urban emergency departments had dedicated trauma teams to care for pediatric trauma patients and providers felt that they were prepared to care for most pediatric trauma patients.

\subsection{Barriers and Challenges Faced by Practitioners in Providing Pediatric Trauma Care}

Barriers to providing optimal pediatric trauma care expressed by providers included lack of pediatric trauma experience, inadequate pediatric trauma training and feeling uncomfortable with initial assessment of the pediatric trauma patient. A rural physician said, "I think we see less of it so we are probably considerably less comfortable with pediatric patients. We just don't encounter it as often.”

Lack of pediatric trauma experience was a consistent theme across all types of providers and hospitals. A regional non-physician provider said, “Generally, we're not overly comfortable with it because we don't do a lot of it. Less than $10 \%$ of our volume is pediatrics to start with. And-and it's so variable and, you know, birth to almost adult. You just can't ever figure out what's supposed to be what. So, I'm going to say nobody would say they're real comfortable with it. They're OK. They do a good job. But as far as that comfort level, no. I don't think it's there overall." One rural non-physician provider also commented, "Well, you know, the- the bad part about it is, 
most of the challenges I think a lot of us face is experience with peds. That is not something that I want to increase to make me more comfortable."

Other challenges identified to providing pediatric trauma care included communication difficulties with the preverbal or crying child, the small size of patients and the emotional aspect of caring for an injured child. An urban physician noted, "It tends to be a little more emotional." An urban nurse stated, "For the staff and also you're dealing with the family members that are a lot higher level of anxiety to try to manage them and try to manage the patient, you're kinda managing the whole family." One rural nurse said, "Things are a little more difficult on the little one, too. I mean, size-wise, it's a smaller area, I mean-You can't put quite as many people working on the patient as you can on an adult. And everybody just seems to be a little more on edge and, you know, a little more chaotic, I guess. And the infrequent part of it, you know? You're just not as ready.” An urban EMS provider commented, "It's difficult to train, at least on the pre-hospital side, how to make decisions on a medical basis and not on an emotional basis when dealing with pediatrics, and I think a lot of our decisions end up being emotional, so it's a lot more stressful.”

\subsection{Training and Education}

Providers in all the hospitals stated that they had the basic class trainings available to them such as Advanced Trauma Life Support (ATLS), Trauma Nursing Core Course (TNCC), Pediatric Advanced Life Support (PALS) but there was a lack pediatric specific training available to the staff, even in large urban hospitals. Overwhelmingly, the providers indicated a need for additional training specific to pediatric trauma. An urban non-physician provider said, "I think increasing that education will kind of combat some of the fear of pediatric traumas."

Most providers across all types of hospitals expressed a need and interest in training focused on pediatric trauma, but topics covered and skills needed varied by type of facility. Rural providers stated they primarily stabilized and prepared patients for transport, so their training needs were in areas related to skills in initial assessment and intervention. One rural physician remarked, "Well, here in pediatric trauma it is primarily stabilization, that's really our focus, beyond that we are pretty quick to move pediatric patients on to situations where we would maximize their chances for survival.”

In contrast, providers at urban hospitals were interested in more in-depth skill development and improving trauma team function, reviewing the latest evidence based recommendations and keeping the trauma team updated on the latest pediatric trauma practices. An urban physician stated, "I would think a lot of what would personally help our docs is what's new in pediatric trauma. Well, what has evidence-based medicine said, in managing, for example, you know, the falls, spinal injuries, and also handling abdominal trauma? From a provider standpoint, those are the things which I would be interested in knowing. You know, what has changed."

The majority of providers in all types of hospitals stated that hands-on training, especially with high-fidelity simulators would be the best way to learn and retain information and skills. One regional physician said, "I think a hands-on is always useful." An urban physician suggested, "I think the more realistic simulation you can get, the-the better the retention is in the training." A similar comment was made by an urban non-physician providers stating, "I have had the benefit of the simulation experience and that is a really powerful learning tool. It was real. I mean, it was as close to real as you could get $\cdots$ so it's a whole different experience that you're able to immerse yourself in. I think it's as close to the real thing as any education can get.” A rural non-physician provider said, "It's (pediatric trauma) so rare we need it. That's what you need to go over, that's what you need to practice because that is what you don't do every day.” A rural physician remarked, "We have to do everything here, yet we're not adequately trained for it."

Another frequent suggestion for training was to make case based scenarios and problem based learning available on interactive web based programs or via telemedicine. For example, a rural non-physician provider suggested, "If you did even one a year that was hands-on but then maybe have an interactive web-based or something to where... everybody can participate. You know, make a web-based interactive and it is open all the time."

\section{Limitations}

Focus groups are designed to examine data based on a selected group' experiences and opinions on a specific topic and may limit generalization to a larger population. Our study was conducted only in community hospitals, so the results may not be generalizable to other emergency departments, particularly academic institutions. Our findings reflect the opinions and perspectives of providers from a single state and certain types of hospitals within that states. Also, since participants are not chosen randomly and the sample group may be small, the potential for selection and information bias exists. Participants who were interested in pediatric trauma care and education may have been more likely to participate which may have led to selection bias in the recruitment of our participants. We did engage participants from a variety of types of institutions to attempt to limit these biases. In general the themes that emerged from one 
group were similar to other groups indicating that the topics were comprehensively covered. Nonetheless, this qualitative study was intended to better understand the experiences and opinions of the participants in order to generate theories and hypothesis that may be tested in further qualitative research studies.

\section{Discussion}

Injury is the leading cause of death in children from one to eighteen years [6]. The severely injured child demands prompt medical intervention for optimal outcome. Since $80 \%$ of children are first cared for by emergency medical services (EMS) and in community hospitals, these health care systems must be prepared [4]. Health system preparation requires necessary personnel, equipment and protocols for initial resuscitation and transfer to definitive care [7]. Practitioner preparation requires competency in pediatric trauma stabilization, encompassing knowledge, skills, and behaviors. Improving care of pediatric trauma patients will require a two-phase approach: one phase to ensure the health system is prepared and another phase to train and improve provider performance. To improve pediatric trauma care first requires a deeper understanding of the issues complicating quality care. In 2001, the American Academy of Pediatrics (AAP) and the American College of Emergency Physicians (ACEP) published "Care of Children in the Emergency Department: Guidelines for Preparedness," which included recommendations for staff training; guidelines for policies, procedures and protocols; support for establishing inter-facility transfer agreements; and an endorsed list of age- and sizeappropriate equipment and supplies [8]. Not all hospitals are prepared to care for children, and not all hospitals furnish the equipment required. A study by Athey et al. [9] in 2001, and a follow up study in 2007 by Burt and Middleton [10], examined discrepancies in pediatric preparedness. Each study demonstrated inconsistencies among hospitals in readiness and equipment. According to the 2008 joint policy statement "Management of Pediatric Trauma [11], "there may also be significant variability in pediatric training and experience among physicians and nurses who staff hospital emergency departments.” The aim of this qualitative study was to understand the barriers and opportunities to pediatric trauma care education specific to the community setting in order to design an education program to improve care.

Optimal pediatric trauma care requires provider knowledge, skills and behaviors that are child-centered. Currently, no national level course is specific to the unique needs of injured children. There are courses that train providers in trauma care including ATLS, Basic Trauma Life Support (BTLS), Pre-Hospital Trauma Life Support (PHTLS), and TNCC. These courses, while they may touch on pediatric specific trauma content (as little as 3\% of ATLS provider training time is devoted to pediatric trauma [12]), are limited in scope and depth. Even pediatric-specific courses such as PALS, Advanced Pediatric Life Support (APLS) and Pediatric Education for Prehospital Providers (PEPP) concentrate primarily on medical emergencies. They provide little rehearsal to adequately prepare providers for injured children. Additionally, up to four years may elapse between re-certifications, which may further challenge care providers' skill and confidence.

Through focus groups conducted in community hospitals participants stated that despite participating in PALS and ATLS they needed more practical focused pediatric training. A recurrent theme expressed by community providers was the lack of enough practical experience to keep their knowledge and skills well rehearsed. Additionally, they identified that training conducted in their practice setting would greatly benefit their overall preparedness. Providers clearly outlined training needs that matched their practice setting and capabilities, which indicated the need for an educational program that is flexible and adaptable to practice setting. Rural providers recognized the need for training and practice that focused on initial stabilization of children rather than definitive care. Regional and urban providers identified need for more in-depth resuscitation assessment and technical skills and team training.

Along with skill matching to practice setting, provider groups also identified the need for interactive practice sessions that were relevant to what they might encounter. They recognized the need to incorporate knowledge of what they need to do with finding the right equipment that was the correct size. They desired to combine the rehearsal of resuscitation technical skills with decisionmaking in team scenarios. These practice sessions should be conducted onsite so they could challenge their preparedness as a team of providers and as a health care setting. A high-fidelity simulation-based training program may be one solution to fulfill these needs.

Simulation-based training as an educational method can recreate and amplify real-life crises with guided and interactive experiences [13]. Likewise, simulation has the benefit of creating situational anxiety and, with it, the optimal level of productive anxiety for learning. Errors can occur without adverse outcomes, which can be more valuable to learning than successes in raising awareness of aspects of performance that need improvement. This attribute allows the teacher/facilitator the opportunity to deconstruct a provider's performance, correct errors and provide feedback until mastery is achieved [14]. Simulation methods commonly employ a strategy of feedback through facilitated debriefing that is based on actual, ob- 
served performance. Rudolph et al. [15] suggest a fourstep model: identifying performance gaps related to predetermined objectives, providing feedback describing the gap, investigating the basis for the gap, and helping to close the gap through discussion and targeted instructions. Several programs have also used simulation to differentiate skill levels of acute care providers in non-trauma applications [16-18].

Pediatric trauma stabilization training has the potential to benefit from simulation training, as major trauma is a high stress, but relatively uncommon disease state. In 2006, Hunt et al. [19] published a study using low fidelity simulation in North Carolina EDs to identify deficiencies in stabilization of children. Common items found to be deficient included accurately estimating a child's weight, obtaining vascular access, removing the child's clothing, offering parental support, performing an accurate neurological assessment and stabilizing the patient's cervical spine. A follow up study by Dr. Hunt [20] using similar simulation and assessment methods, found that scenario participation with a brief educational intervention was effective in improving performance in follow up testing. Significant overall trauma team performance improvement was shown in a study comparing scenarios conducted in the first 4 months of the study year versus scenarios conducted during the last 4 months of the year following team-based pediatric simulation training at Cincinnati Children's Hospital [21]. A recent report by the IOM [4] stated that EDs "must ensure that their workforce is well prepared to handle pediatric patients." The report went on to suggest that simulation "should be used for continuing education in order to provide as realistic event as possible" to allow rehearsal and team skill training. An adult, learner-focused, adaptable program that trains providers with the knowledge, skills, and behaviors specific to children is needed to address this IOM concern. Such a training program also needs to include a method to evaluate and correct deficiencies in the health care environment in addition to the individual practitioner. Assessment methods that have undergone validity and reliability testing are currently missing, however. This deficiency was noted as a top priority in a consensus report published by the Society of Academic Emergency Medicine [22].

\section{Conclusion}

In summary, results from focus groups conducted in community hospitals revealed that all community emergency room providers indicated a need for pediatric trauma education. Specifically, hands-on training with highfidelity simulation was identified as the most useful type of training to gain the skills and confidence needed to manage pediatric trauma patients in their emergency de- partments. Results from this qualitative study will help form the basis of future studies for the development of pediatric trauma simulation training programs.

\section{Acknowledgements}

This study was funded by the Center for Excellence in Pediatric Resuscitation. We would like to thank the coordinator for Kansas Area Health Education Centers, Mary Beth Warren, RN for her help with recruitment of participants and Amy Altergott, RN for her participation in the focus group activities.

\section{REFERENCES}

[1] Centers for Disease Control and Prevention, "Injury Prevention and Control: Data and Statistics (WISQARS)," Nonfatal Injury Data, 2010.

www.cdc.gov/injury/wisqars/index.html

[2] M. L. Nance, B. G. Carr and C. C. Branas, "Access to Pediatric Trauma Care in the United States," Archives of Pediatrics and Adolescent Medicine, Vol. 163, No. 6, 2009, pp. 512-518. http://dx.doi.org/10.1001/archpediatrics.2009.65

[3] American College of Emergency Physicians Policy Resource and Education Paper, The Pediatric Emergency Medicine Committee, "Report on Preparedness of the Emergency Department for the Care of Children,” 2009. www.acep.org/content.aspx?id=30140

[4] Committee on the Future of Emergency Care in the United States Health System, Institute of Medicine of the National Academies, "Emergency Care for Children: Growing Pains,” The National Academies Press, Washington DC, 2006.

[5] B. Hancock, E. Ockleford and K. Windridge, "An Introduction to Qualitative Research,” Trent RDSU, 2007.

[6] Centers for Disease Control and Prevention, National Center for Health Statistics, VitalStats, 2010. http://www.cdc.gov/nchs/vitalstats.htm

[7] J. S. Seidel, M. Hornbein, K. Yoshiyama, et al., "Emergency Medical Services and the Pediatric Patient: Are the Needs Being Met?” Pediatrics, Vol. 73, No. 6, 1984, pp. 769-772.

[8] American Academy of Pediatrics, Committee on Pediatric Emergency Medicine and American College of Emergency Physicians, Pediatric Care Committee, "Care of Children in the Emergency Department: Guidelines for Preparedness," Pediatrics, Vol. 107, No. 4, 2001, pp. 777-781. http://dx.doi.org/10.1542/peds.107.4.777

[9] J. Athey, J. M. Dean, J. Ball, et al., “Ability of Hospitals to Care for Pediatric Emergency Patients," Pediatric Emergency Care, Vol. 17, No. 3, 2001, pp. 170-174. http://dx.doi.org/10.1097/00006565-200106000-00005

[10] C. W. Burt and K. R. Middleton, "Factors Associated with Ability to Treat Pediatric Emergencies in US Hospitals,” Pediatric Emergency Care, Vol. 23, No. 10, 2007, pp. 681-689. 
http://dx.doi.org/10.1097/PEC.0b013e3181558d43

[11] American Academy of Pediatrics Section on Orthopedics, Committee of Pediatric Emergency Medicine, Section on Critical Care, Section on Surgery, Section on Transport Medicine and Pediatric Orthopedic Society of North America, "Management of Pediatric Trauma," Pediatrics, Vol. 121, No. 4, 2008, pp. 849-854.

[12] Advanced Trauma Life Support Course Syllabus.

[13] D. M. Gaba, "The Future Vision of Simulation in Healthcare,” Quality \& Safety in Health Care, Vol. 13, Suppl. 1, 2004, pp. i2-i10.

http://dx.doi.org/10.1136/qshc.2004.009878

[14] K. A. Ericsson, "Deliberate Practice and Acquisition of Expert Performance: A General Review,” Academic Emergency Medicine, Vol. 15, No. 11, 2008, pp. 988-994. http://dx.doi.org/10.1111/j.1553-2712.2008.00227.x

[15] J. W. Rudolph, R. Simon, D. B. Raemer, et al., "Debriefing as Formative Assessment: Closing the Performance Gaps in Medical Education,” Academic Emergency Medicine, Vol. 15, No. 11, 2008, pp. 1010-1016. http://dx.doi.org/10.1111/j.1553-2712.2008.00248.x

[16] D. V. Girzadas Jr., L. Clay, J. Caris, et al., "High Fidelity Simulation Can Discriminate between Novice and Experienced Residents When Assessing Competency in Patient Care,” Medical Teacher, Vol. 29, No. 5, 2007, pp. 452-456. http://dx.doi.org/10.1080/01421590701513698

[17] F. L. Overly, S. N. Sudikoff and M. J. Shapiro, "HighFidelity Medical Simulation as an Assessment Tool for
Pediatric Residents' Airway Management Skills,” Pediatric Emergency Care, Vol. 23, No. 1, 2007, pp. 11-15. http://dx.doi.org/10.1097/PEC.0b013e31802c61d5

[18] M. B. Brett-Fleeger, R. J. Vinci, D. L. Weiner, et al., “A Simulator-Based Tool That Assesses Pediatric Residents' Resuscitation Competency,” Pediatrics, Vol. 121, No. 3, 2008, pp. e597-e603. http://dx.doi.org/10.1542/peds.2005-1259

[19] E. A. Hunt, S. M. Hohenhaus, X. Luo, et al., "Simulation of Pediatric Trauma Stabilization in 35 North Carolina Emergency Departments: Identification of Targets for Performance Improvement," Pediatrics, Vol. 117, No. 3, 2006, pp. 641-648. http://dx.doi.org/10.1542/peds.2004-2702

[20] E. A. Hunt, M. Heine, S. M. Hohenhaus, et al., "Simulated Pediatric Trauma Team Management: Assessment of an Educational Intervention," Pediatric Emergency Care, Vol. 23, No. 11, 2007, pp. 796-804. http://dx.doi.org/10.1097/PEC.0b013e31815a0653

[21] R. A. Falcone Jr., M. Daugherty, L. Schweer, et al., "Multidisciplinary Pediatric Trauma Team Training Using High-Fidelity Simulation,” Journal of Pediatric Surgery, Vol. 43, No. 6, 2008, pp. 1065-1071. http://dx.doi.org/10.1016/j.jpedsurg.2008.02.033

[22] "Proceedings of the 2008 AEM Consensus Conference: The Science of Simulation in Healthcare: Defining and Developing Clinical Experience,” Academic Emergency Medicine, Vol. 15, No. 11, 2008, pp. 978-1214. 